\title{
Colloidal and Antibacterial Properties of Novel Triple-Headed, Double-Tailed Amphiphiles: Exploring Structure-Activity Relationships and Synergistic Mixtures
}

John N. Marafino a,b, Tara M. Gallagher ${ }^{\text {a }}$, Jhosdyn Barragan ${ }^{\text {b }}$, Brandi L. Volkers ${ }^{\text {a }}$, Jade E. LaDow ${ }^{\text {a }}$, Kyle Bonifer ${ }^{\mathrm{a}}$, Gabriel Fitzgerald ${ }^{\mathrm{b}}$, Jason L. Floyd ${ }^{\mathrm{a}}$, Kristin McKenna ${ }^{\mathrm{b}}$, Nicholas T. Minahan ${ }^{\mathrm{a}}$, Brenna Walsh ${ }^{\mathrm{b}}$, Kyle Seifert ${ }^{\mathrm{a}, * *}$ Kevin L. Caran ${ }^{\mathrm{b}, *}$

a James Madison University, Department of Biology, 951 Carrier Drive, MSC 7801, Harrisonburg, VA 22807, USA

b James Madison University, Department of Chemistry and Biochemistry, 901 Carrier Drive, MSC 4501, Harrisonburg, VA 22807, USA

Abbreviations: MBC, Minimum Bactericidal Concentration; MIC, Minimum

Inhibitory Concentration; CAC, Critical Aggregation Concentration; ARI,

Antimicrobial-Resistant Infections; ITC, Isothermal Titration Calorimetry.

* Corresponding author. Tel.: 1540568 6632; fax: 15405687938.

E-mail address: carankl@jmu.edu (K.L. Caran).

** Corresponding author. Tel.: 1540568 2286; fax: 15405683333.

E-mail address: seiferkx@jmu.edu (K. Seifert).

Both corresponding authors contributed equally to this work.

\section{Abstract:}

Two novel series of tris-cationic, tripled-headed, double-tailed amphiphiles were synthesized and the effects of tail length and head group composition on the critical aggregation concentration (CAC), thermodynamic parameters, and minimum inhibitory concentration (MIC) against six bacterial strains were investigated. Synergistic antibacterial combinations of these amphiphiles were also identified. Amphiphiles in this study are composed of a benzene core with three benzylic ammonium bromide groups, two of which have alkyl chains, each 8 to 16 carbons in length. The third head group is a trimethylammonium or pyridinium. Log of critical aggregation concentration $(\log [\mathrm{CAC}])$ and heat of aggregation $\left(\Delta H_{\mathrm{agg}}\right)$ were both inversely proportional to the length of the linear hydrocarbon chains. Antibacterial activity increases with tail length until an optimal tail length of 12 carbons per chain, above which, activity decreased. The derivatives with two 12 carbon chains had the best antibacterial activity, killing all tested strains at concentrations of 1-2 $\mu \mathrm{M}$ for Gram-positive and 4-16 $\mu \mathrm{M}$ for Gram-negative bacteria. The identity of the third head group (trimethylammonium or pyridinium) had minimal effect on colloidal and antibacterial activity. The antibacterial activity of several binary combinations of amphiphiles from this study was higher than activity of individual amphiphiles, indicating that these combinations are synergistic. These amphiphiles show promise as novel antibacterial agents that could be used in a variety of applications.

\section{Introduction}

Over the past few decades, the overuse of antibiotics has resulted in an increase in antibiotic-resistant bacteria. ${ }^{1-5}$ In addition, the production of novel antimicrobials continues to decrease, primarily due to low financial return. ${ }^{5,6}$ This combination of circumstances has contributed to the increasing prevalence of antimicrobial-resistant infections (ARIs), especially in the hospital setting. ARIs have contributed to tens of 
thousands of deaths per year in the European Union and United States alone. ${ }^{7,8}$ Hospitals and nursing homes are particularly prone to harboring antimicrobial-resistant organisms due to the frequent use of antimicrobial agents and an influx of infected, and frequently, immunocompromised patients. ${ }^{9}$ Limiting the transmission of bacteria from contaminated equipment and individuals to a susceptible population is critical to reducing the risk of hospital acquired infections by antibiotic resistant organisms. ${ }^{9}{ }^{10}$ The development of effective novel antibacterials could be of benefit in the healthcare setting by decreasing the cost associated with treating infected patients, and ultimately, decreasing mortality due to these types of infections. ${ }^{11}$

The use of cationic amphiphiles as antimicrobials was first reported in $1935 .{ }^{12}$ Amphiphiles continue to be utilized as antimicrobial agents in detergents, disinfectants, cosmetics, and other common household products. ${ }^{13}$ A large variety of novel amphiphiles has been synthesized in an effort to increase effectiveness and specificity. ${ }^{14-24}$

Amphiphile structure, including size and relative number of hydrophobic tails and hydrophilic head groups, governs colloidal characteristics including the critical aggregation concentration (CAC) and thermodynamic properties. ${ }^{25}$ At concentrations below the $\mathrm{CAC}$, amphiphiles tend to align at the air-water interface in equilibrium with dissolved monomers in solution. ${ }^{25,26}$ At concentrations above the CAC, amphiphiles form aggregates in which hydrocarbon tails interact with each other releasing water that was formerly associated with the tails, resulting in a second equilibrium between monomers and aggregates in solution. ${ }^{25,27}$ An increase in entropy typically associated with aggregate formation is generally attributed in large part to the release of water molecules surrounding hydrophobic units to the bulk water that accompanies this process. ${ }^{25}$ Increasing amphiphile hydrophobicity (for example by increasing the length or number of tails) decreases water solubility, thus decreasing CAC. In contrast, additional head groups can increase amphiphile solubility, typically resulting in a higher CAC. ${ }^{28-31}$

Amphiphile structure also affects antimicrobial effectiveness. A trend reported by multiple studies is the relationship between amphiphile tail length and the minimum inhibitory concentration (MIC), the lowest concentration at which an antimicrobial is able to inhibit microbial growth. Typically, as tail length increases the MIC decreases until an optimal tail length is reached. The MIC then increases for amphiphiles with tail lengths exceeding this optimal length. ${ }^{14-21}$ Variations in spacing between head groups can also impact the MIC. For example, studies conducted by our labs show that amphiphiles with a 5-carbon spacer between two cationic head groups are the most biologically active. ${ }^{14}$ The amphiphiles in the current study also have a 5-carbon spacer between cationic groups.

Some mixtures of two or more amphiphiles exhibit synergy - the inhibition of bacteria at lower concentrations than when each amphiphile is used separately. ${ }^{32}$ By decreasing the required concentration of 
compounds, combination therapy reduces the potential for, or degree of, adverse side effects and increases the effectiveness of antibacterial agents. The improved efficacy of synergistic combinations has contributed to the improvement of hand disinfectants and effective treatment for patients with ARIs. ${ }^{33-36}$

In this report, the synthesis and colloidal, antibacterial, and synergistic characteristics for two novel series of triple-headed, double-tailed amphiphiles are reported (Fig. 1). Amphiphiles in this study consist of three cationic head groups connected to a mesitylene $(M)$ core. Two of the head groups are dimethylalkylammonium groups with linear hydrocarbon tails from 8 to 16 carbons in length. The third head group is either pyridinium ( $M-P$ series) or trimethylammonium ( $M-1$ series).

\section{Results and Discussion}

\subsection{Synthesis}

Each of the amphiphiles in this study was prepared by two subsequent Menshutkin reactions (Scheme 1). To prepare the $M$-P series (1-5), 1,3,5-trisbromomethylbenezene was reacted with a slight excess of pyridine resulting in intermediate $\mathbf{1 1}$ (77\% yield). Selective reaction at just one of the three equivalent benzylic positions was aided by the decreased solubility of the desired product, which precipitates from the reaction mixture upon formation. Filtration and washing with acetone provides compound $\mathbf{1 1}$ in sufficient purity for the next synthetic step. Substitution of the two remaining benzylic bromides on $\mathbf{1 1}$ was accomplished using excess dimethylalkylamine $\left(\mathrm{NMe}_{2}\left(\mathrm{CH}_{2}\right)_{\mathrm{n}-1} \mathrm{CH}_{3}\right.$, where $\mathrm{n}=8,10,12,14$ or 16) in ethanol at reflux producing the $M-P$ series of amphiphilic products $1-5$ (63-92\% yield).

To prepare the $M-1$ series (6-10), 1,3,5 trisbromomethylbenezene was reacted with a slight excess of trimethylamine resulting in intermediate $\mathbf{1 2}$ (37\% yield). This reaction produces primarily $\mathbf{1 2}$, along with a highly insoluble biscationic byproduct (a result of two benzylic bromides being substituted with trimethylamine), which can be effectively removed by an extraction protocol as detailed in the experimental section. Substitution of the two remaining benzylic bromides on $\mathbf{1 2}$ was accomplished using excess dimethylalkylamine $\left(\mathrm{NMe}_{2}\left(\mathrm{CH}_{2}\right)_{\mathrm{n}-1} \mathrm{CH}_{3}\right.$, where $\left.\mathrm{n}=8,10,12,14,16\right)$ in ethanol at reflux producing the $M-1$ series of amphiphilic products 6-10 (20 - 75\% yield).

\subsection{Critical Aggregation Concentration and Heat of Aggregate Formation}

Isothermal titration calorimetry (ITC) was used to determine the critical aggregation concentration (CAC) and the thermodynamic parameters associated with aggregate formation for each amphiphile (Table 1). Briefly, the Nano ITC was used to measure the heat change associated with the deaggregation of amphiphiles via power compensation. ${ }^{37-40}$ A concentrated aqueous solution of amphiphile (>>CAC) was titrated into a thermally controlled sample cell, initially containing pure water, in a series of discrete injections. Typical ITC results can be divided into three ranges associated with the concentration of 
amphiphile in the sample cell below, at, and above the CAC, as seen for compound 9 (M-1,14,14, Fig. 2 ). During initial injections (Range I), measured heat changes are due to both the dilution of aggregates and deaggregation. This can be observed in the first 10 injections, where aggregates absorbed heat and disassociated into dissolved monomers. ${ }^{\text {a }}$ The heat change produced by each injection was approximately constant in this range. In Range II, injected aggregates don't fully dissociate, reducing the total absorbed heat for each subsequent injection. At concentrations well above the CAC (Range III), absorbed heat is attributed only to the dilution of aggregates and is approximately constant. The CAC was determined by the inflection point in Range II, as measured by the minimum value in the plot of CAC versus the first derivative of the heat (Fig. 2, inset). Analogous results were recorded for compounds 2-5 and 7-10, the results of which are presented in table 1. CAC and thermodynamic data were not determined for the amphiphiles with the shortest tails $[\mathbf{1}(M-P, 8,8)$ and $\mathbf{6}(M-1,8,8)]$ because the heat evolved during injection was above the threshold of the instrument.

Both series of compounds exhibit a decrease in CAC as tail length increases (Table 1). CAC values for the $M-P$ and $M-1$ series are approximately equivalent for each tail length, following a linear plot of $\log (\mathrm{CAC})$ versus tail length (Fig. 3a) according to the equation:

$$
\log (\mathrm{CAC})=A-B n
$$

where $A$ and $B$ are constants and $n$ is the number of carbons in each hydrocarbon tail. ${ }^{29,41,42}$ The dependence of CAC on tail length is the same for each series $(B=0.31)$.

The difference between the average heat of injections in Range I, representing the total heat due to the dilution of aggregates and deaggregation, and the average heat of injections in Range III, representing the heat due solely to the dilution of aggregates, gives the heat of deaggregation, $\Delta H_{\text {deagg. }}$. The $\Delta H_{\text {deagg }}$ for compound $9(M-1,14,14)$ is $37 \mathrm{~kJ} / \mathrm{mol}$, corresponding to a heat of aggregate formation, $\Delta H_{\text {agg }}$, of $-37 \mathrm{~kJ} / \mathrm{mol}$ $\left(\Delta H_{\text {agg }}=-\Delta H_{\text {deagg }}\right.$ ). The negative $\Delta H_{\text {agg }}$ values indicate that aggregation is an exothermic process for all of the amphiphiles in the study (Table 1). This is attributed to the release of heat accompanying aggregate formation from additional hydrogen bonding of water molecules joining the bulk, and from hydrocarbon tail interactions in the newly formed aggregate. Furthermore, $\Delta H_{\text {agg }}$ becomes more negative as tail length increases for both series of amphiphiles, following a linear trend (Fig. 3b) according to the equation:

$$
\Delta H_{\mathrm{agg}}=C-D n
$$

\footnotetext{
a Typically the $1^{\text {st }}$ injection is ignored. ${ }^{43}$
} 
where $C$ and $D$ are constants and $n$ is the number of carbons in each hydrocarbon tail. ${ }^{29,41,42}$ The dependence of $\Delta H_{\mathrm{agg}}$ on tail length is similar for each series $(D=-5.1$ for the $M-P$ series; $D=-5.8$ for the $M-1$ series). The inverse relationship between $\Delta H_{\text {agg }}$ and amphiphile tail length is consistent with a larger amount of water released per molecule of amphiphile, as well as greater van der Waals interactions between the longer tails.

The $\Delta G_{\text {agg }}$ can be approximated for the aggregation of nonionic amphiphiles by the equation

$$
\Delta G_{\mathrm{agg}}=R T \ln (\mathrm{CAC} / 55.5)
$$

where the $\mathrm{CAC}$ represents an equilibrium between monomeric and aggregated amphiphiles. ${ }^{43,44}$ The CAC is expressed as a molar fraction [molar units divided by the molar concentration of water $(55.5 \mathrm{~mol} / \mathrm{L})]$. This equation changes for ionic amphiphiles due to the presence of counterions and the degree of ionization at the aggregate surface. Thus the equation is modified for an ionic amphiphile as ${ }^{43,44}$

$$
\Delta G_{\text {agg }}=R T[1+(m / n)] \ln (\mathrm{CAC} / 55.5)
$$

where $m$ is the concentration of counterions that associate with a aggregate and $n$ is the number of monomers that associate to form an aggregate. ${ }^{43,44}$ Since this study did not include determination of the degree of ionization or aggregate aggregation, $\Delta G_{\text {agg }}$ was approximated using equation (3), as has been done by others. ${ }^{28,39,40}$ The $\Delta G_{\text {agg }}$ for all compounds in the study is negative and becomes more negative as tail length increases (Table 1). Thus the increase in chain length within a series of amphiphiles is consistent with the increased propensity to spontaneously form aggregates.

The entropy of aggregation ( $\Delta S_{\text {agg }}$ ) is dictated by a combination of entropic factors that favor or disfavor aggregate formation and can be approximated using the Gibbs-Helmholtz equation:

$$
\Delta G_{\mathrm{agg}}=\Delta H_{\mathrm{agg}}-T \Delta S_{\mathrm{agg}}
$$

To aid the reader, the entropic contribution to $\Delta G_{\text {agg }}$, is presented as the negative of $T \Delta S_{\text {agg }}$ (Table 1). In both series, the $-T \Delta S_{\text {agg }}$ term is negative for amphiphiles with chain lengths of up to 12 carbons [ 2 ( $M$ $P, 10,10), \mathbf{3}(M-P, 12,12), 7(M-1,10,10)$, and $\mathbf{8}(M-1,12,12)]$. This suggests that the entropy gained from the release of water from hydrophobic sections is greater than the decrease in entropy of amphiphiles due to the formation of aggregates. Thus the entropic factor, for amphiphiles with shorter chain lengths, contributes to the spontaneous formation (negative $\Delta G_{\text {agg }}$ ) of aggregates. 
The $-T \Delta S_{\text {agg }}$ term is positive for double tailed amphiphiles with tail lengths exceeding 12 carbons [4 ( $M$ $P, 14,14), \mathbf{5}(M-P, 16,16), \mathbf{9}(M-1,14,14)$, and $\mathbf{1 0}(M-1,16,16)]$. This suggests that the entropy gained from the release of water from hydrophobic units is smaller than the decrease in entropy of amphiphiles due to the formation of aggregates. Thus amphiphiles with longer chains entropically disfavor formation of aggregates making aggregate formation of these derivatives solely an enthalpy-driven process. This decrease in entropy may be explained by considering the effect of an amphiphile's cone angle on aggregate size. ${ }^{42}$ As tail length increases in a series of analogous amphiphiles, the cone angle decreases resulting in a higher aggregation number per aggregate. Formation of larger aggregates results in a larger decrease in entropy upon aggregate formation due to the greater number of amphiphiles per aggregate.

\subsection{Minimum Inhibitory Concentration}

The MIC values of all compounds from both series were determined for four Gram-positive (Staphylococcus aureus, Enterococcus faecalis, Streptococcus agalactiae, and Bacillus subtilis) and two Gram-negative (Escherichia coli and Pseudomonas aeruginosa) strains (Table 2). For both series, the MIC decreases with tail length until an optimal tail length of 12 carbons per chain, above which the MIC increases (Fig. 4). The derivatives with two 12-carbon chains, compounds $3(M-P, 12,12)$ and $\mathbf{8}(M$ $1,12,12)$, have the lowest MIC values against each strain with values ranging from 1-2 $\mu \mathrm{M}$ for Grampositive bacteria and 4-16 $\mu \mathrm{M}$ for Gram-negative bacteria. This trend is indicative of the relationship between solubility and bioactivity and is consistent with previous studies on other amphiphile series. ${ }^{14,15,19}$ Higher MIC values against Gram-negative strains may be due to their outer membrane, which is not present in Gram-positive bacteria.

Many antibacterial agents are ineffective against $P$. aeruginosa due to its semipermeable outer membrane and production of efflux pumps and $\beta$-lactamases. ${ }^{45}$ The contamination of medical equipment with $P$. aeruginosa biofilms contributes to hospital-acquired infections, particularly caused by antibiotic-resistant strains. ${ }^{46-50}$ While other antibacterial agents fail to inhibit $P$. aeruginosa, two amphiphiles in this study [3 $(M-P, 12,12)$ and $\mathbf{8}(M-1,12,12)]$ kill this organism at relatively low concentrations, which may prove useful in a healthcare setting. Notably, MIC values of compounds $\mathbf{3}(M-P, 12,12)$ and $\mathbf{8}(M-1,12,12)$ against $P$. aeruginosa ( $8 \mu \mathrm{M}$ and $16 \mu \mathrm{M}$, respectively) are comparable to those of tobramycin $(6.4 \mu \mathrm{M})$, commonly used to treat infection in cystic fibrosis patients, and cefepime $(12.5 \mu \mathrm{M})$, an antispeduomonal cephalosporin. ${ }^{51,52}$

MIC values between respective $M-P$ and $M-1$ tail lengths were generally similar, indicating that replacing the trimethylammonium head group with a pyridinium did not significantly affect bioactivity. A notable exception is observed between the two derivatives with 16-carbon chains, where the trimethylammonium derivative $(\mathbf{1 0}, M-1,16,16)$ was more effective than the pyridinium derivative $(\mathbf{5}, M-P, 16,16)$ for all strains except $P$. aeruginosa. For all tested compounds, the MBC was the same concentration or a two-fold 
concentration higher than the MIC, indicating the amphiphiles are bactericidal, consistent with previous studies on other multiheaded amphiphiles. ${ }^{14,53}$

With only a few exceptions, MIC values are significantly below CAC values, demonstrating that amphiphile aggregation is not required to kill bacteria. ${ }^{19}$ At concentrations near or above the CAC the amphiphile may act as a detergent, solubilizing the cell membrane - a mechanism of action that could be detrimental to prokaryotic cells. ${ }^{17,19}$ Amphiphiles at sub-CAC levels are potent antibacterials at concentrations where detergent effects are not observed. It is recognized CAC values reported here (measured in pure water) may differ to some degree from the CAC values in the medium used for MIC studies.

\subsection{Synergistic combinations}

In addition to determining the antibacterial activity of individual amphiphiles, combination studies were performed in order to determine if binary mixtures of amphiphiles demonstrate synergy in killing representative Gram-positive (S. aureus) and Gram-negative (E. coli) organisms (Fig. 5). A combination is considered synergistic if a mixture of amphiphiles has greater antimicrobial activity than simply an additive effect. The fractional inhibitory concentration (FIC) was used to indicate whether two chemicals were synergistic (FIC $<0.5$ ), indifferent (FIC 0.5-4.0), or antagonistic (FIC >4.0). ${ }^{32}$ Two amphiphile combinations are synergistic against $S$. aureus: combinations of compounds $\mathbf{3}$ and $\mathbf{4}(\mathrm{FIC}=0.31)$ and compounds $\mathbf{3}$ and $\mathbf{1 0}$ ( $\mathrm{FIC}=0.38$ ). In addition, one combination is synergistic against $E$. coli: compound $\mathbf{3}$ and $\mathbf{9}$ (FIC=0.38). All three synergistic combinations contain compound $\mathbf{3}(M-P, 12,12)$. The use of synergistic antibacterial combinations could be beneficial in a health-care setting, where frequent antimicrobial use has contributed to the presence of antibiotic-resistant organisms. ${ }^{33,34,36,54-57}$

\section{Conclusions}

Two novel series of amphiphiles were synthesized and structure-activity relationships were investigated. $\log (\mathrm{CAC})$ and $\Delta H_{\text {agg }}$ each decrease linearly with increasing tail length for both series. For most of the amphiphiles in this study, the MIC is significantly below the CAC, indicating amphiphile aggregation is not necessary for antibacterial activity. MIC values also indicated an optimal tail length of 12 carbons for each series as compounds $\mathbf{3}$ and $\mathbf{8}(M-P, 12,12$ and $M-1,12,12)$ have the lowest MIC against all strains tested. Furthermore, three binary combinations of amphiphiles have been identified that exhibit synergistic relationships. These amphiphiles could prove useful in the medical field especially when treating or preventing infection with antibiotic-resistant organisms.

\section{Methods and Materials}




\subsection{Synthesis}

Synthesis of $\mathbf{1 1}$

1,3,5-tribromomethylbenezene (1.0 g, $2.8 \mathrm{mmol}$, Sigma-Aldrich) was dissolved in acetone (200 $\mathrm{mL})$ at room temperature in a round bottom flask. The solution was equipped with a stir-bar and attached to an addition funnel. Pyridine (0.45 mL, $5.6 \mathrm{mmol}$, Sigma-Aldrich) was dissolved in acetone (100 $\mathrm{mL})$ and added drop-wise to the stirring solution of 1,3,5-tribromethylbenzene at room temperature and allowed to react overnight. A white precipitate formed. The crude reaction mixture was vacuum filtered, washed with acetone, and dried under vacuum yielding $943 \mathrm{mg}$ (77\%) of a tan solid, 11. See Supporting Information for analytical data.

\section{Synthesis of 12}

1,3,5-tribromomethylbenezene $(2.01 \mathrm{~g}, 5.6 \mathrm{mmol})$ was dissolved in acetone $(400 \mathrm{~mL})$ at room temperature in a round bottom flask. The solution was cooled on an ice bath for 30 minutes, equipped with a stir-bar, and attached to an addition funnel. An ethanolic trimethylamine solution ( $1.6 \mathrm{~mL}, 6.7 \mathrm{mmol}$, ACROS $)$ was diluted with acetone $(200 \mathrm{~mL})$ and cooled on an ice bath for 30 minutes. The trimethylamine solution was added drop-wise to the stirring solution of 1,3,5-tribromethylbenzene. The reaction was run overnight, and allowed to warm slowly to room temperature. A white precipitate containing a mixture of $\mathbf{1 2}$ and a biscationic side product formed. The crude reaction mixture was briefly heated to $50^{\circ} \mathrm{C}$ and vacuum filtered. The mother liquor (which contained a mixture of $\mathbf{1 2}$ and unreacted starting material) was transfered to a clean RBF and the solvent was removed by rotary evaporation. The resulting solid was resuspended in room temperature acetone for 30 minutes, vacuum filtered, and dried under vacuum yielding 12 (white solid). The solid filtered from the crude reaction mixture was resuspended in a solution of acetone and ethanol (100:3), heated to $60{ }^{\circ} \mathrm{C}$, stirred for at least 30 minutes, and vacuum filtered. The filtrate was transferred to a clean flask and the solvent was removed by rotary evaporation yielding additional 20 (white solid, total yield $=860 \mathrm{mg}, 37 \%$ ). See Supporting Information for analytical data.

\section{Synthesis of 1-10: General Protocol A}

Compound 11 or $\mathbf{1 2}$ was added to a two-neck round bottom flask and dissolved in ethanol. The flask was equipped with a stir bar and attached to a water-cooled condenser. Excess dimethylalkylamine $\left(\mathrm{NMe}_{2}\left(\mathrm{CH}_{2}\right)_{\mathrm{n}-1} \mathrm{CH}_{3}\right.$, where $\mathrm{n}=8,10,12,14$ or 16) was added slowly to the flask via syringe. The reaction was heated to reflux and allowed react overnight. Volatile materials were removed under a flow of $\mathrm{N}_{2}(\mathrm{~g})$. The resulting crude solid was resuspended in acetone to dissolve any residual dimethyalkylamine and vacuum filtered to yield the solid amphiphile product (1-10). Details for each product and analytical data are provided in the Supporting Information.

\subsection{Isothermal Titration Calorimetry}


$\mathrm{CAC}$ and $\Delta H_{\text {agg }}$ were determined using a Nano-ITC (TA-Instruments). Prior to each experiment the sample cell was washed with $\mathrm{dH}_{2} \mathrm{O}(300 \mathrm{~mL})$, ethanol $(100 \mathrm{~mL}), \mathrm{dH}_{2} \mathrm{O}(300 \mathrm{~mL})$ and nanopure water $(200 \mathrm{~mL})$. Next, $950 \mu \mathrm{L}$ of nanopure water was added to the sample cell. A concentrated aqueous solution ( $>\mathrm{CAC}$ ) of amphiphile was prepared and equilibrated at $37^{\circ} \mathrm{C}$. A $250 \mu \mathrm{L}$ syringe was filled with the aqueous solution, and loaded into the Nano ITC. Multiple single injections in aliquots of $5 \mu \mathrm{L}$ were injected into the sample cell with time intervals varying from 300s to 1400s. Samples were continuously stirred (300 rpm) throughout the titration. The Nano-Analyze program (TA-Instruments) was used to analyze the data. CAC and $\Delta H_{\text {agg }}$ values reported are the average of two or more repeat experiments for each amphiphile.

\subsection{Bacterial strains and growth conditions}

The Gram-positive bacterial strains used in this study were Staphylococcus aureus subsp. aureus ATCC® $29213^{\mathrm{TM}}$, Enterococcus faecalis ATCC® $29212^{\mathrm{TM}}$, Bacillus subtilis, and Streptococcus agalactiae $\mathrm{J} 48 .^{58}$ The Gram-negative bacterial strains used were Escherichia coli ATCC® $25922^{\mathrm{TM}}$ and Pseudomonas aeruginosa ATCC ${ }^{2} 27853^{\mathrm{TM}}$. All strains were grown in $1 \mathrm{X}$ Mueller-Hinton Broth at $37^{\circ} \mathrm{C}$ for 12-24 $\mathrm{h}$. For the MIC and combination studies, bacterial suspensions were prepared by diluting overnight cultures to $5 \times 10^{6} \mathrm{CFU} / \mathrm{mL}$ in $2 \mathrm{X}$ Mueller-Hinton Broth, so that when amphiphile solutions are added the final broth strength is $1 \mathrm{X}$.

\subsection{Minimum Inhibitory Concentration and Minimum Bactericidal Concentration}

The methods used to determine the MIC and MBC were performed as previously described and followed the standards set forth by the Clinical and Laboratory Standards Institute. ${ }^{14,59}$ Briefly, compounds were serially diluted and $100 \mu \mathrm{L}$ of each dilution were added to the wells of a 96-well flat-bottomed microtiter plate in triplicate. After adding $100 \mu \mathrm{L}$ of the bacterial cell suspension, the plates were incubated at $37^{\circ} \mathrm{C}$ for $72 \mathrm{~h}$. The MIC of the compound was defined as the minimum concentration that resulted in visible inhibition of bacterial growth. In order to determine the $\mathrm{MBC}$, a $100 \mu \mathrm{l}$ aliquot from each triplicate well was grown on Todd-Hewitt agar and incubated for $24 \mathrm{~h}$ at $37^{\circ} \mathrm{C}$. The $\mathrm{MBC}$ was defined as the concentration of the compound that resulted in a $99.9 \%$ reduction of the bacterial CFU/mL. The MIC was considered to be bactericidal if the MBC was the same concentration or one concentration higher in the dilution series as the MIC. ${ }^{53}$

\subsection{Combination Studies}

To determine if two amphiphiles act synergistically to kill E. coli or $S$. aureus, combination studies were performed using the checkerboard technique as described. ${ }^{32}$ Amphiphiles with MIC values higher than the maximum concentration used (>250 $\mu \mathrm{M}$ ) were excluded from the combination studies. The amphiphile concentrations used in the combination studies ranged from 1/16 to $2 \mathrm{x}$ the MIC. Fifty microliters of each amphiphile dilution and $100 \mu \mathrm{L}$ of the bacterial suspension were added to the wells of a 96-well flatbottomed microtiter plate. Control wells consisted of the bacterial suspension treated with media alone and 
bacteria treated with individual amphiphiles. Plates were incubated at $37{ }^{\circ} \mathrm{C}$ for $72 \mathrm{~h}$. The FIC index was calculated using the following formula: $\mathrm{FIC}=\mathrm{FIC}_{\mathrm{A}}+\mathrm{FIC}_{\mathrm{B}}=\mathrm{A} / \mathrm{MIC}_{\mathrm{A}}+\mathrm{B} / \mathrm{MIC}$. $\mathrm{A}$ and $\mathrm{B}$ are the $\mathrm{MIC}$ values of compound $\mathrm{A}$ and compound $\mathrm{B}$ when combined, and $\mathrm{MIC}_{\mathrm{A}}$ and $\mathrm{MIC}_{\mathrm{B}}$ are the $\mathrm{MIC}$ of compound $\mathrm{A}$ and $\mathrm{B}$ alone. ${ }^{32} \mathrm{~A}$ combination was considered to be synergistic if the FIC was less than 0.5; a combination was considered indifferent with an FIC of 0.5-4; and a combination was considered antagonistic with an FIC > 4. The FIC indices of synergistic combinations were confirmed in two separate experiments.

\section{Acknowledgement}

We would like to thank Dr. Louise Temple for donating B. subtilis, Drs. Chris Berndson and Nathan Wright for assistance with ITC, Dr. Christine Hughey for assistance with the TOF-HRMS, Drs. Yanjie Zhang and Thomas DeVore for valuable discussions regarding the treatment of thermodynamic data and

Drs. Daniel Ralston and Jun Yin for technical assistance. This work was supported by a Multi-Investigator Cottrell College Science Award from the Research Corporation for Science Advancement (MICCSA 10709), the National Science Foundation (NSF-REU CHE-1062629 and CHE-0754521), the James Madison University College of Science and Mathematics (including Faculty Assistance Grants to KS and $\mathrm{KC}$ and additional support for students and supplies), and the JMU Departments of Biology and Chemistry and Biochemistry. Funding from two NSF MRI grants (CHE-0922935 and CHE-1046630) was used to purchase the TOF MS instrument used in exact mass measurements. 


\section{References}

1. Fan, F. F. Defining and Combating the Mechanisms of Triclosan Resistance in Clinical Isolates of Staphylococcus aureus. Antimicrob. Agents Chemother. 2002, 46, 3343-3347.

2. Chea, P. Executive summary: Select findings, conclusions, and policy recommendations. Clin. Infect. Dis. 2005, 41, S224-S227.

3. Gilbert, M.; MacDonald, J.; Louie, M.; Gregson, D.; Zhang, K.; Elsayed, S.; Laupland, K.; Nielsen, D.; Wheeler, V.; Lye, T.; Conly, J. Prevalence of USA300 colonization or infection and associated variables during an outbreak of community-associated methicillin-resistant Staphylococcus aureus in a marginalized urban population. Can. J. Infect. Dis. Med. 2007, 18, 357-362.

4. Okeke, I. N.; Lamikanra, A.; Edelman, R. Socioeconomic and behavioral factors leading to acquired bacterial resistance to antibiotics in developing countries. Emerg. Infect. Dis. 1999, 5, 18-27.

5. Taubes, G. The bacteria fight back. Science. 2008, 321, 356-360+361.

6. Scheffler, R. J.; Colmer, S.; Tynan, H.; Demain, A. L.; Gullo, V. P. Antimicrobials, drug discovery, and genome mining. Appl. Microbiol. Biotechnol. 2013, 97, 969-978.

7. WHO Evolving threat of antimicrobial resistance. Who. Drug. Inf. 2012, 26, 125-125.

8. CDC Antibiotic Resistance Threats in the United States, 2013. Medical Benefits. 2014, 31, 12-12.

9. Johnston, B. L.; Bryce, E. Hospital infection control strategies for vancomycin-resistant Enterococcus, methicillin-resistant Staphylococcus aureus and Clostridium difficile. Can. Med. Assoc. J. 2009, 180, 627-631.

10. Goldmann, D. A.; Weinstein, R. A.; Wenzel, R. P.; Tablan, O. C.; Duma, R. J.; Gaynes, R. P.; Schlosser, J.; Martone, W. J. Strategies to prevent and control the emergence and spread of antimicrobial-resistant microorganisms in hospitals: A challenge to hospital leadership. J. Am. Med. Assoc. 1996, 275, 234-240.

11. MacDonald, A.; Dinah, F.; MacKenzie, D.; Wilson, A. Performance feedback of hand hygiene, using alcohol gel as the skin decontaminant, reduces the number of inpatients newly affected by MRSA and antibiotic costs. J. Hosp. Infect. 2004, 56, 56-63. 
12. Gerhard, D. A new class of disinfectants. Dtsch med Wochenschr. 1935, 61, 829-832.

13. Annual Review of Cosmetic Ingredient Safety Assessments: 2005/2006 Int. J. Toxicol. 2008, 27, 77142.

14. Ladow, J. E.; Warnock, D. C.; Hamill, K. M.; Simmons, K. L.; Davis, R. W.; Schwantes, C. R.; Flaherty, D. C.; Willcox, J. A. L.; Wilson-Henjum, K.; Caran, K. L.; Minbiole, K. P. C.; Seifert, K. Bicephalic amphiphile architecture affects antibacterial activity. Eur. J. Med. Chem. 2011, 46, 42194226.

15. Grenier, M. C.; Davis, R. W.; Wilson-Henjum, K. L.; LaDow, J. E.; Black, J. W.; Caran, K. L.; Seifert, K.; Minbiole, K. P. C. The antibacterial activity of 4,4'-bipyridinium amphiphiles with conventional, bicephalic and gemini architectures. Bioorg. Med. Chem. Lett. 2012, 22, 4055-4058.

16. Maisuria, B. B.; Actis, M. L.; Hardrict, S. N.; Falkinham III, J. O.; Cole, M. F.; Cihlar, R. L.; Peters, S. M.; Macri, R. V.; Sugandhi, E. W.; Williams, A. A.; Poppe, M. A.; Esker, A. R.; Gandour, R. D. Comparing micellar, hemolytic, and antibacterial properties of di- and tricarboxyl dendritic amphiphiles. Bioorg. Med. Chem. 2011, 19, 2918-2926.

17. Macri, R. V.; Karlovská, J.; Doncel, G. F.; Du, X.; Maisuria, B. B.; Williams, A. A.; Sugandhi, E. W.; Falkinham III, J. O.; Esker, A. R.; Gandour, R. D. Comparing anti-HIV, antibacterial, antifungal, micellar, and cytotoxic properties of tricarboxylato dendritic amphiphiles. Bioorg. Med. Chem. 2009, $17,3162-3168$.

18. Sugandhi, E. W.; Falkinham III, J. O.; Gandour, R. D. Synthesis and antimicrobial activity of symmetrical two-tailed dendritic tricarboxylato amphiphiles. Bioorg. Med. Chem. 2007, 15, 38423853.

19. Sugandhi, E. W.; Macri, R. V.; Williams, A. A.; Kite, B. L.; Slebodnick, C.; Falkinham,J.O.; Esker, A. R.; Gandour, R. D. Synthesis, critical micelle concentrations, and antimycobacterial properties of homologous, dendritic amphiphiles. Probing intrinsic activity and the "cutoff" effect. J. Med. Chem. 2007, 50, 1645-1650.

20. Williams, A. A.; Sugandhi, E. W.; Macri, R. V.; Falkinham, J. O.; Gandour, R. D. Antimicrobial activity of long-chain, water-soluble, dendritic tricarboxylato amphiphiles. J. Antimicrob. Chemoth. 2007, 59, 451-458.

21. Falkinham III, J. O.; Macri, R. V.; Maisuria, B. B.; Actis, M. L.; Sugandhi, E. W.; Williams, A. A.; Snyder, A. V.; Jackson, F. R.; Poppe, M. A.; Chen, L.; Ganesh, K.; Gandour, R. D. Antibacterial 
activities of dendritic amphiphiles against nontuberculous mycobacteria. Tuberculosis. 2012, 92, 173181.

22. Ator, L. E.; Jennings, M. C.; McGettigan, A. R.; Paul, J. J.; Wuest, W. M.; Minbiole, K. P. C. Beyond paraquats: dialkyl 3,3'- and 3,4'-bipyridinium amphiphiles as antibacterial agents. Bioorg. Med. Chem. Lett. 2014, 24, 3706-3709.

23. Black, J. W.; Jennings, M. C.; Azarewicz, J.; Paniak, T. J.; Grenier, M. C.; Wuest, W. M.; Minbiole, K. P. C. TMEDA-derived biscationic amphiphiles: An economical preparation of potent antibacterial agents. Bioorg. Med. Chem. Lett. 2014, 24, 99-102.

24. Haldar, J.; Kondaiah, P.; Bhattacharya, S. Synthesis and antibacterial properties of novel hydrolyzable cationic amphiphiles. Incorporation of multiple head groups leads to impressive antibacterial activity. J. Med. Chem. 2005, 48, 3823-3831.

25. Scamehorn, J. F.; Sabatini, D. A.; Harwell, J. H. Surfactants, Part I: Fundamentals. In Encyclopedia of Supramolecular ChemistryMarcel Dekker: New York, 2004; pp 1458.

26. Pohorille, A.; Deamer, D. Self-assembly and function of primitive cell membranes. Res. Microbiol. 2009, 160, 449-456.

27. Soontravanich, S.; Munoz, J. A.; Scamehorn, J. F.; Harwell, J. H.; Sabatini, D. A. Interaction between an anionic and an amphoteric surfactant. Part I: Monomer-micelle equilibrium. J. Surfactants. Deterg. 2008, 11, 251-261.

28. Bhattacharya, S.; Haldar, J. Microcalorimetric and Conductivity Studies with Micelles Prepared from Multi-Headed Pyridinium Surfactants†े. Langmuir. 2005, 21, 5747-5751.

29. Roszak, K. Z.; Torcivia, S. L.; Hamill, K. M.; Hill, A. R.; Radloff, K. R.; Crizer, D. M.; Middleton, A. M.; Caran, K. L. Biscationic bicephalic (double-headed) amphiphiles with an aromatic spacer and a single hydrophobic tail. J. Colloid Interface Sci. 2009, 331, 560-564.

30. Haldar, J.; Aswal, V. K.; Goyal, P. S.; Bhattacharya, S. Molecular Modulation of Surfactant Aggregation in Water: Effect of the Incorporation of Multiple Headgroups on Micellar Properties. Angewandte Chemie International Edition. 2001, 40, 1228-1232.

31. Bhattacharya, S.; Samanta, S. K. Surfactants Possessing Multiple Polar Heads. A Perspective on their Unique Aggregation Behavior and Applications. J. Phys. Chem. Lett. 2011, 2, 914-920. 
32. Giacometti, A.; Cirioni, O.; Del Prete, M. S.; Paggi, A. M.; D’Errico, M. M.; Scalise, G. Combination studies between polycationic peptides and clinically used antibiotics against Gram-positive and Gram-negative bacteria. Peptides. 2000, 21, 1155-1160.

33. Geraldo, I. M.; Gilman, A.; Shintre, M. S.; Modak, S. M. Rapid antibacterial activity of 2 novel hand soaps: evaluation of the risk of development of bacterial resistance to the antibacterial agents. Infect. Control Hosp. Epidemiol. 2008, 29, 736-741.

34. Hiraki, Y.; Yoshida, M.; Masuda, Y.; Inoue, D.; Tsuji, Y.; Kamimura, H.; Karube, Y.; Takaki, K.; Kawano, F. Successful treatment of skin and soft tissue infection due to carbapenem-resistant Acinetobacter baumannii by ampicillin-sulbactam and meropenem combination therapy. Int. J. Infect. Dis. 2013, 17, e1234-e1236.

35. Sick, A. C.; Tschudin-Sutter, S.; Turnbull, A. E.; Weissman, S. J.; Tamma, P. D. Empiric combination therapy for Gram-negative bacteremia. Pediatrics. 2014.

36. Daikos, G. L.; Petrikkos, P.; Psichogiou, M.; Kosmidis, C.; Vryonis, E.; Skoutelis, A.; Georgousi, K.; Tzouvelekis, L. S.; Tassios, P. T.; Bamia, C.; Petrikkos, G. Prospective observational study of the impact of VIM-1 metallo-beta-lactamase on the outcome of patients with Klebsiella pneumoniae bloodstream infections. Antimicrob. Agents Chemother. 2009, 53, 1868-1873.

37. Wiseman, T.; Williston, S.; Brandts, J. F.; Lin, L. N. Rapid measurement of binding constants and heats of binding using a new titration calorimeter. Anal. Biochem. 1989, 179, 131-137.

38. Kresheck, G. C.; Hargraves, W. A. Thermometric titration studies of the effect of head group, chain length, solvent, and temperature on the thermodynamics of micelle formation. J. Colloid Interface Sci. 1974, 48, 481-493.

39. Paula, S.; Süs, W.; Tuchtenhagen, J.; Blume, A. Thermodynamics of micelle formation as a function of temperature: A high sensitivity titration calorimetry study. J. Phys. Chem. 1995, 99, 11742-11751.

40. Heerklotz, H.; Seelig, J. Titration calorimetry of surfactant-membrane partitioning and membrane solubilization. BBA-Biomembranes. 2000, 1508, 69-85.

41. Rosen, M. J. Surfactants and Interfacial Phenomena. 2004, Third Edition.

42. Israelachvili, J. Fluid-Like Structure and Self-Assembling Systems: Micelles, Bilayers and Biological Membranes. In Intermolecular and Surface ForcesAcademic Press INC: San Diego, CA, 1991; pp 341 . 
43. Myers, D. Classic Thermodynamics of Micelle Formation. In Surface, Interfaces and Colloids Principles and Applications, Second Edition ed.;John Wiley \& Sons: 1999; pp 369.

44. Rosen, M. J. Thermodynamic Parameters of Micellization. In Surfactants and Interfacial Phenomena, Third Edition ed.;John Wiley \& Sons: Hoboken, New Jersey, 2004; pp 161.

45. Gellatly, S. L.; Hancock, R. E. W. Pseudomonas aeruginosa: new insights into pathogenesis and host defenses. Pathog Dis. 2013, 67, 159-173.

46. Hallin, M.; Deplano, A.; Roisin, S.; Boyart, V.; De Ryck, R.; Nonhoff, C.; Byl, B.; Glupczynski, Y.; Denis, O. Pseudo-outbreak of extremely drug-resistant Pseudomonas aeruginosa urinary tract infections due to contamination of an automated urine analyzer. J. Clin. Microbiol. 2012, 50, 580582.

47. Romano, S.; Bourdier, A.; Parer, S.; Masnou, A.; Burgel, L.; Raczka, F.; Lamy, B.; Jumas-Bilak, E.; Lotthé, A. Peripheral venous catheter and bloodstream infection caused by Pseudomonas aeruginosa after a contaminated preoperative shower. Infect. Control Hosp. Epidemiol. 2013, 34, 544-546.

48. Lanini, S.; D'Arezzo, S.; Puro, V.; Martini, L.; Imperi, F.; Piselli, P.; Montanaro, M.; Paoletti, S.; Visca, P.; Ippolito, G. Molecular epidemiology of a Pseudomonas aeruginosa hospital outbreak driven by a contaminated disinfectant-soap dispenser. PLOS ONE. 2011, 6.

49. Kohlenberg, A.; Weitzel-Kage, D.; van der Linden, P.; Sohr, D.; Vögeler, S.; Kola, A.; Halle, E.; Rüden, H.; Weist, K. Outbreak of carbapenem-resistant Pseudomonas aeruginosa infection in a surgical intensive care unit. J. Hosp. Infect. 2010, 74, 350-357.

50. Carlos A. DiazGranados, M.; Marolyn Y. Jones, R.; Thiphasone Kongphet-Tran, B.; Nancy White, R.; Mark Shapiro, M.; Yun F. Wang, P.; Susan M. Ray, M.; Henry M. Blumberg, M. Outbreak of Pseudomonas aeruginosa infection associated with contamination of a flexible bronchoscope. Infection Control and Hospital Epidemiology. 2009, 30, 550-555.

51. Meyer, R. D.; Young, L. S.; Armstrong, D. Tobramycin (nebramycin factor 6): in vitro activity against Pseudomonas aeruginosa. Appl. Microbiol. 1971, 22, 1147-1151.

52. Bodey, G. P.; Ho, D. H.; LeBlanc, B. In vitro studies of BMY-28142, a new broad-spectrum cephalosporin. Antimicrob. Agents Chemother. 1985, 27, 265-269.

53. Motyl, M.; Dorso, K.; Barrett, J.; Giacobbe, R. Basic microbiological techniques used in antibacterial drug discovery. Curr. Protoc. Pharmacol. 2006, Chapter 13. 
54. Lister, P. D.; Wolter, D. J. Levofloxacin-imipenem combination prevents the emergence of resistance among clinical isolates of Pseudomonas aeruginosa. Clinical Infectious Diseases. 2005, 40, S105S114.

55. Gribble, M. J.; Chow, A. W.; Naiman, S. C.; Smith, J. A.; Bowie, W. R.; Sacks, S. L.; Grossman, L.; Buskard, N.; Growe, G. H.; Plenderleith, L. H. Prospective randomized trial of piperacillin monotherapy versus carboxypenicillin-aminoglycoside combination regimens in the empirical treatment of serious bacterial infections. Antimicrob. Agents Chemother. 1983, 24, 388-393.

56. Drew, K. R. P.; Sanders, L. K.; Culumber, Z. W.; Zribi, O.; Wong, G. C. L. Cationic amphiphiles increase activity of aminoglycoside antibiotic tobramycin in the presence of airway polyelectrolytes. J. Am. Chem. Soc. 2009, 131, 486-493.

57. Harrison, J. J.; Turner, R. J.; Joo, D. A.; Stan, M. A.; Chan, C. S.; Allan, N. D.; Vrionis, H. A.; Olson, M. E.; Ceri, H. Copper and quaternary ammonium cations exert synergistic bactericidal and antibiofilm activity against Pseudomonas aeruginosa. Antimicrob. Agents Chemother. 2008, 52, 2870-2881.

58. Seifert, K. N.; McArthur, W. P.; Bleiweis, A. S.; Brady, L. J. Characterization of group B streptococcal glyceraldehyde-3-phosphate dehydrogenase: surface localization, enzymatic activity, and proteinprotein interactions. Can. J. Microbiol. 2003, 49, 350-356.

59. P. A. Wayne Methods for dilution antimicrobial tests for bacteria that grow aerobically. 2009. 
Figure 1. Structures of triple-headed, double-tailed amphiphiles in this study. Compounds are named as follows: $M-X, n, n$ where $M$ refers to the Mesitylene core, and $X$ and $n$ represent the attached groups: $P$ indicates a pyridinium; a number indicates the number of carbon atoms in the alkyl group of a dimethylalkyl ammonium (e.g., $l=$ trimethylammonium; $8=$ octyldimethylammonium).

Scheme 1. Synthesis of triple-headed, double-tailed amphiphiles.

Figure 2. Isothermal titration calorimetry was used to determine the critical aggregation concentration (CAC) and $\Delta H_{\text {agg. }}$ a) The heat rate associated with each injection versus time for $\mathbf{9}(M-1,14,14)$. b) Integration of the heat per injection normalized by the number of moles of each injection versus the increasing concentration of amphiphile in the well. Inset: CAC is determined by calculating the first derivative the heat curve.

Figure 3. Plots of $\log (\mathrm{CAC})$ (a) and $\Delta H_{\text {agg }}$ (b) versus tail length at $37^{\circ} \mathrm{C}$ for the $M-P(\mathbf{O})$ and $M-1$ series.

Figure 4. Effect of tail length and head group on $\log (\mathrm{MIC})(M-P$ series $=\mathbf{0} ; M-1$ series $=\mathbf{\square})$. \# $=\mathrm{MIC}>$ $250 \mu \mathrm{M} . \mathrm{G}^{+}=$Gram-positive; $\mathrm{G}^{-}=$Gram-negative .

Figure 5. Synergy studies for $M-P$ and $M-1$ amphiphiles tested against $E$. coli and $S$. aureus. The FIC value for each combination is given. Shaded blocks indicate synergistic combinations (FIC <0.5). Numbers below the diagonal represent FIC values against $S$. aureus. Numbers above the diagonal represent FIC values against $E$. coli. 
Table 1. Critical aggregation concentration (CAC) and thermodynamic parameters at $37^{\circ} \mathrm{C}$ for the $M-P$ and $M-1$ series.

\begin{tabular}{|c|c|c|c|c|c|c|c|c|c|c|}
\hline \multirow{2}{*}{$M-P$} & \multirow{2}{*}{$M-1$} & \multirow{2}{*}{$\begin{array}{c}\text { Tail Length } \\
(n \text { for } M-X, n, n)\end{array}$} & \multicolumn{2}{|c|}{$\mathbf{C A C}(\mathrm{mM})$} & \multicolumn{2}{|c|}{$\Delta \boldsymbol{H}_{\text {agg }}(\mathrm{kJ} / \mathrm{mol})$} & \multicolumn{2}{|c|}{$\Delta \boldsymbol{G}_{\mathrm{agg}}(\mathrm{kJ} / \mathrm{mol})$} & \multicolumn{2}{|c|}{$\boldsymbol{- T} \Delta \boldsymbol{S}_{\mathrm{agg}}(\mathrm{kJ} / \mathrm{mol})$} \\
\hline & & & $M-P$ & $M-1$ & $M-P$ & $M-1$ & $M-P$ & $M-1$ & $M-P$ & $M-1$ \\
\hline 2 & 7 & 10,10 & 14 & 12 & -11 & -15 & -21 & -22 & -10 & -6.6 \\
\hline 3 & 8 & 12,12 & 2.4 & 2.5 & -23 & -26 & -26 & -26 & -3.1 & -0.082 \\
\hline 4 & 9 & 14,14 & 0.60 & 0.61 & -34 & -37 & -29 & -29 & 4.9 & 8.0 \\
\hline 5 & 10 & 16,16 & 0.18 & 0.16 & -42 & -55 & -33 & -33 & 9.2 & 22 \\
\hline
\end{tabular}


Table 2. MIC values $(\mu \mathrm{M})$ for $M-P$ and $M-1$ amphiphiles. $\mathrm{G}^{+}=$Gram-positive, $\mathrm{G}^{-}=$Gram-negative.

\begin{tabular}{|c|c|c|c|c|c|c|c|c|c|c|c|c|c|c|}
\hline \multirow[t]{2}{*}{$M-P$} & \multirow[t]{2}{*}{$M-1$} & \multirow{2}{*}{$\begin{array}{c}\text { Tail length } \\
(n \text { for } M-X, n, n)\end{array}$} & \multicolumn{2}{|c|}{$\begin{array}{l}\text { B. subtilis } \\
\left(\mathrm{G}^{+}\right)\end{array}$} & \multicolumn{2}{|c|}{$\begin{array}{c}\text { E. faecalis } \\
\qquad\left(\mathrm{G}^{+}\right)\end{array}$} & \multicolumn{2}{|c|}{$\begin{array}{l}\text { S. agalactiae } \\
\qquad\left(\mathrm{G}^{+}\right)\end{array}$} & \multicolumn{2}{|c|}{$\begin{array}{l}\text { S. aureus } \\
\left(\mathrm{G}^{+}\right)\end{array}$} & \multicolumn{2}{|c|}{$\begin{array}{c}\text { E. coli } \\
\left(\mathrm{G}^{-}\right)\end{array}$} & \multicolumn{2}{|c|}{$\begin{array}{c}\text { P. aeruginosa } \\
\left(\mathrm{G}^{-}\right)\end{array}$} \\
\hline & & & $M-P$ & $M-1$ & $M-P$ & $M-1$ & $M-P$ & $M-1$ & $M-P$ & $M-1$ & $M-P$ & $M-1$ & $M-P$ & $M-1$ \\
\hline 1 & 6 & 8,8 & $>250$ & $>250$ & $>250$ & $>250$ & $>250$ & $>250$ & $>250$ & $>250$ & $>250$ & $>250$ & $>250$ & $>250$ \\
\hline 2 & 7 & 10,10 & 8 & 8 & 8 & 8 & 8 & 4 & 2 & 16 & 16 & 125 & 63 & $>250$ \\
\hline 3 & 8 & 12,12 & 2 & 2 & 2 & 2 & 1 & 2 & 2 & 2 & 4 & $4-8$ & 8 & 16 \\
\hline 4 & 9 & 14,14 & 8 & 4 & 4 & 4 & 4 & $2-4$ & 4 & $4-8$ & 16 & 8 & 63 & 125 \\
\hline 5 & 10 & 16,16 & $>250$ & 16 & $>250$ & 32 & $>250$ & 8 & $>250$ & 16 & $>250$ & 63 & $>250$ & $>250$ \\
\hline
\end{tabular}




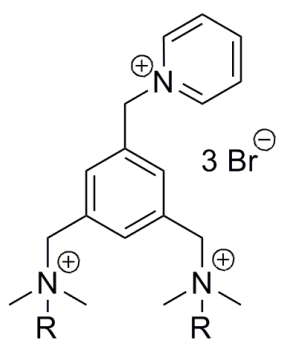

1, $\mathrm{R}=\mathrm{C}_{8} \mathrm{H}_{17}(M-P, 8,8)$

2, $\mathrm{R}=\mathrm{C}_{10} \mathrm{H}_{21}(M-P, 10,10)$

3, $\mathrm{R}=\mathrm{C}_{12} \mathrm{H}_{25}(M-P, 12,12)$

4, $\mathrm{R}=\mathrm{C}_{14} \mathrm{H}_{29}(M-P, 14,14)$

5, $\mathrm{R}=\mathrm{C}_{16} \mathrm{H}_{33}(M-P, 16,16)$

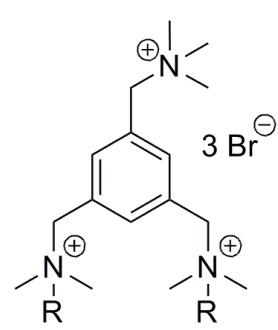

6, $\mathrm{R}=\mathrm{C}_{8} \mathrm{H}_{17}(M-1,8,8)$

7, $\mathrm{R}=\mathrm{C}_{10} \mathrm{H}_{21}(M-1,10,10)$

8, $\mathrm{R}=\mathrm{C}_{12} \mathrm{H}_{25}(M-1,12,12)$

9, $\mathrm{R}=\mathrm{C}_{14} \mathrm{H}_{29}(M-1,14,14)$

10, $\mathrm{R}=\mathrm{C}_{16} \mathrm{H}_{33}(M-1,16,16)$ 


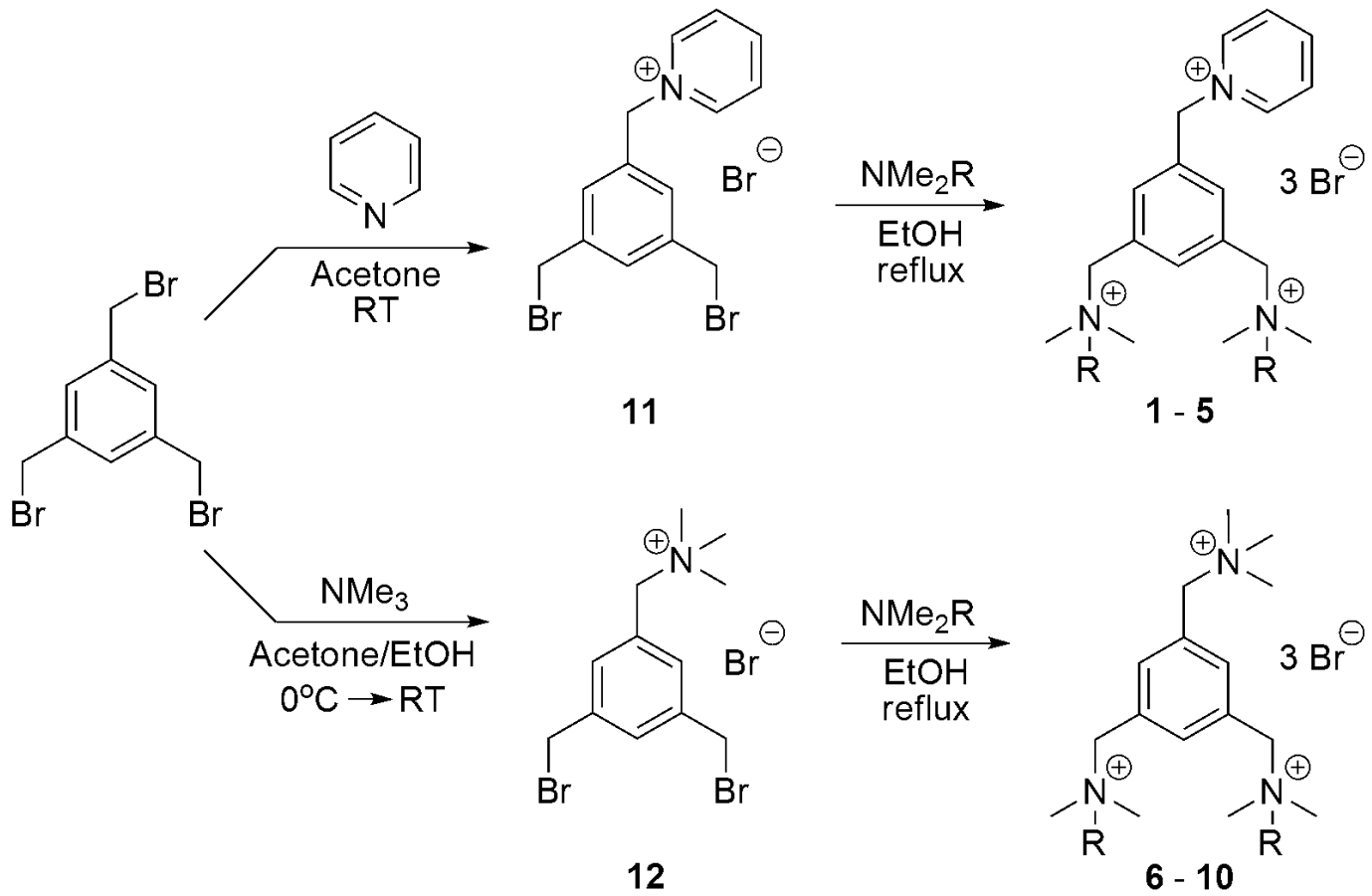



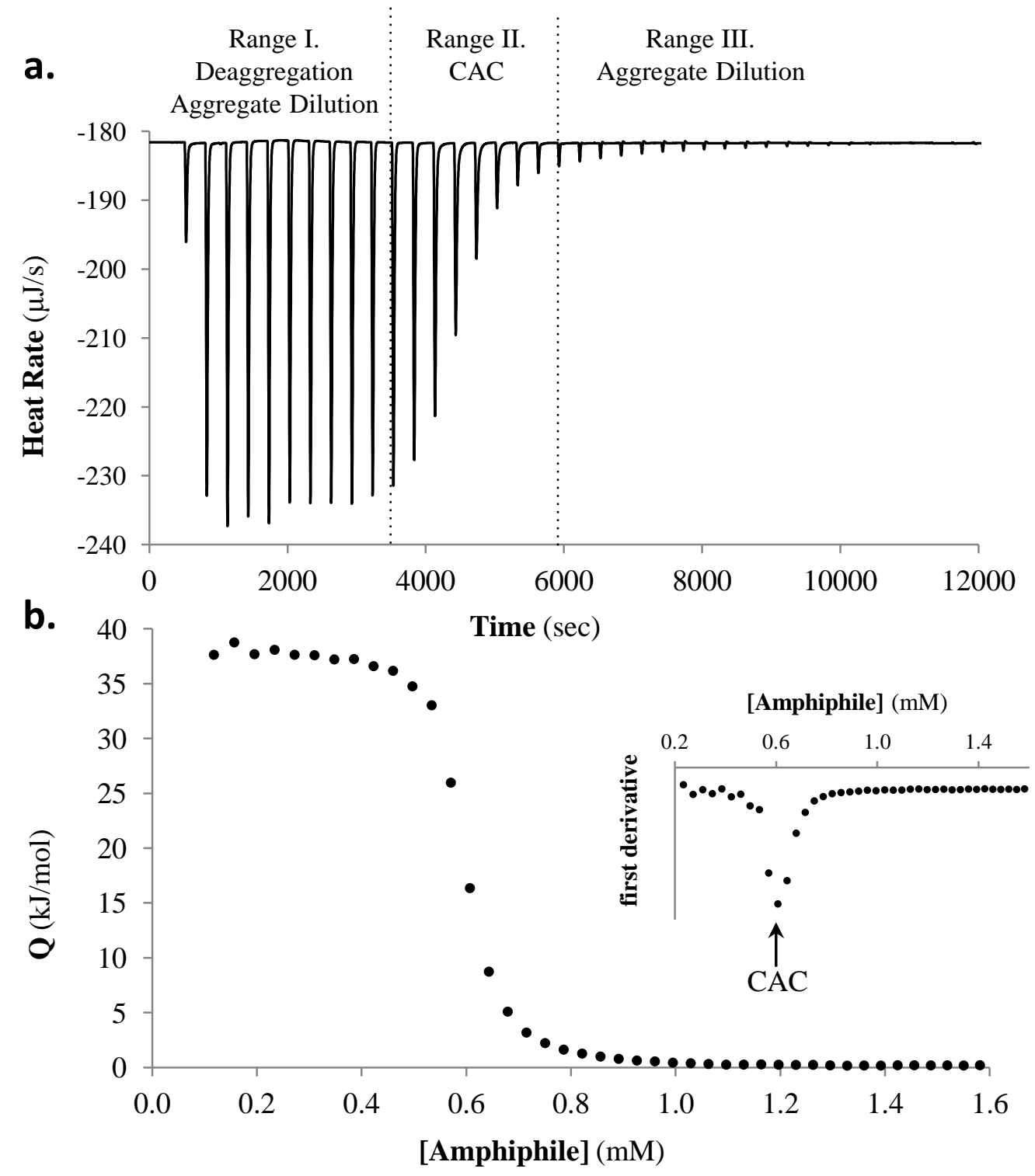

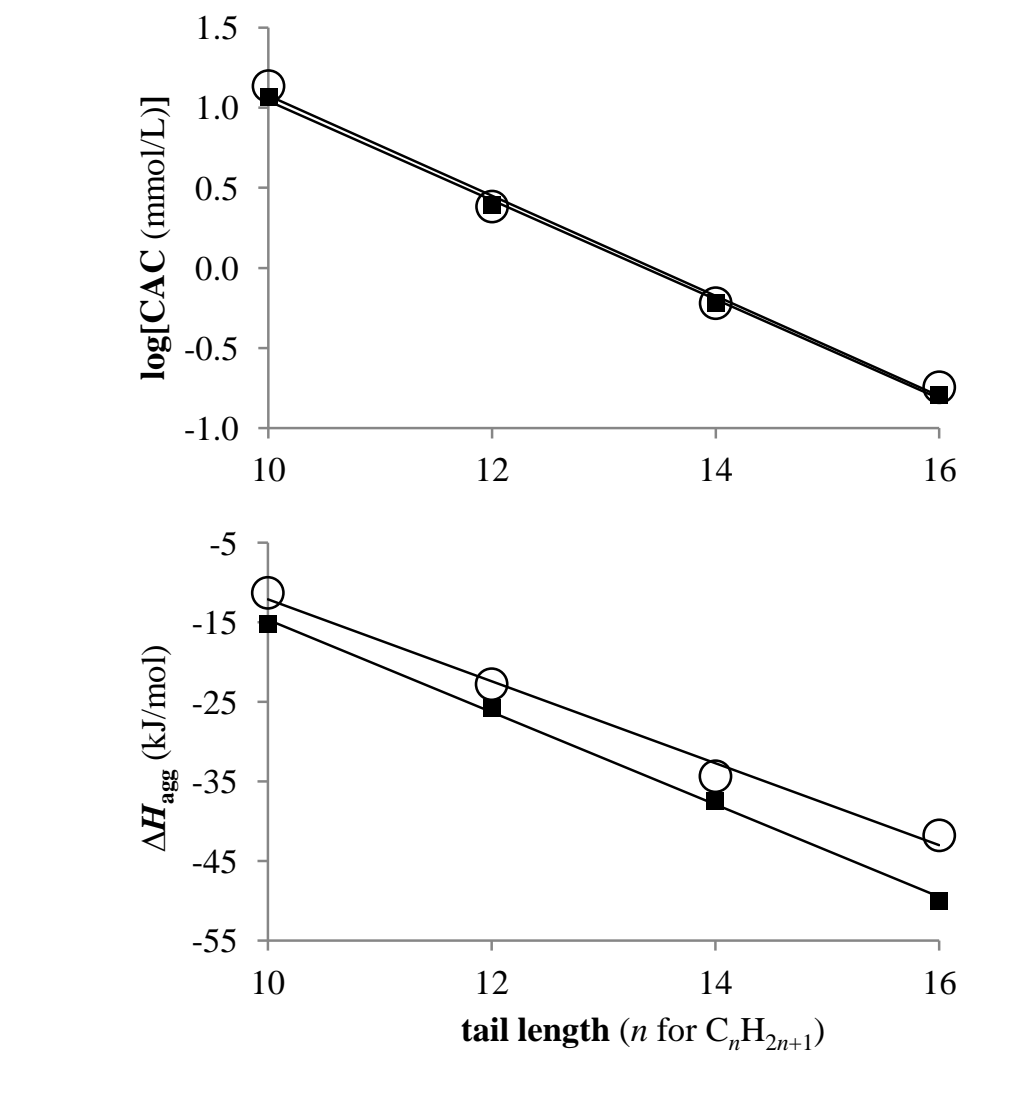

Figure 3

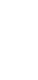

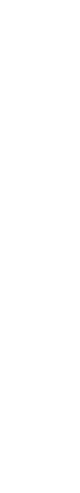



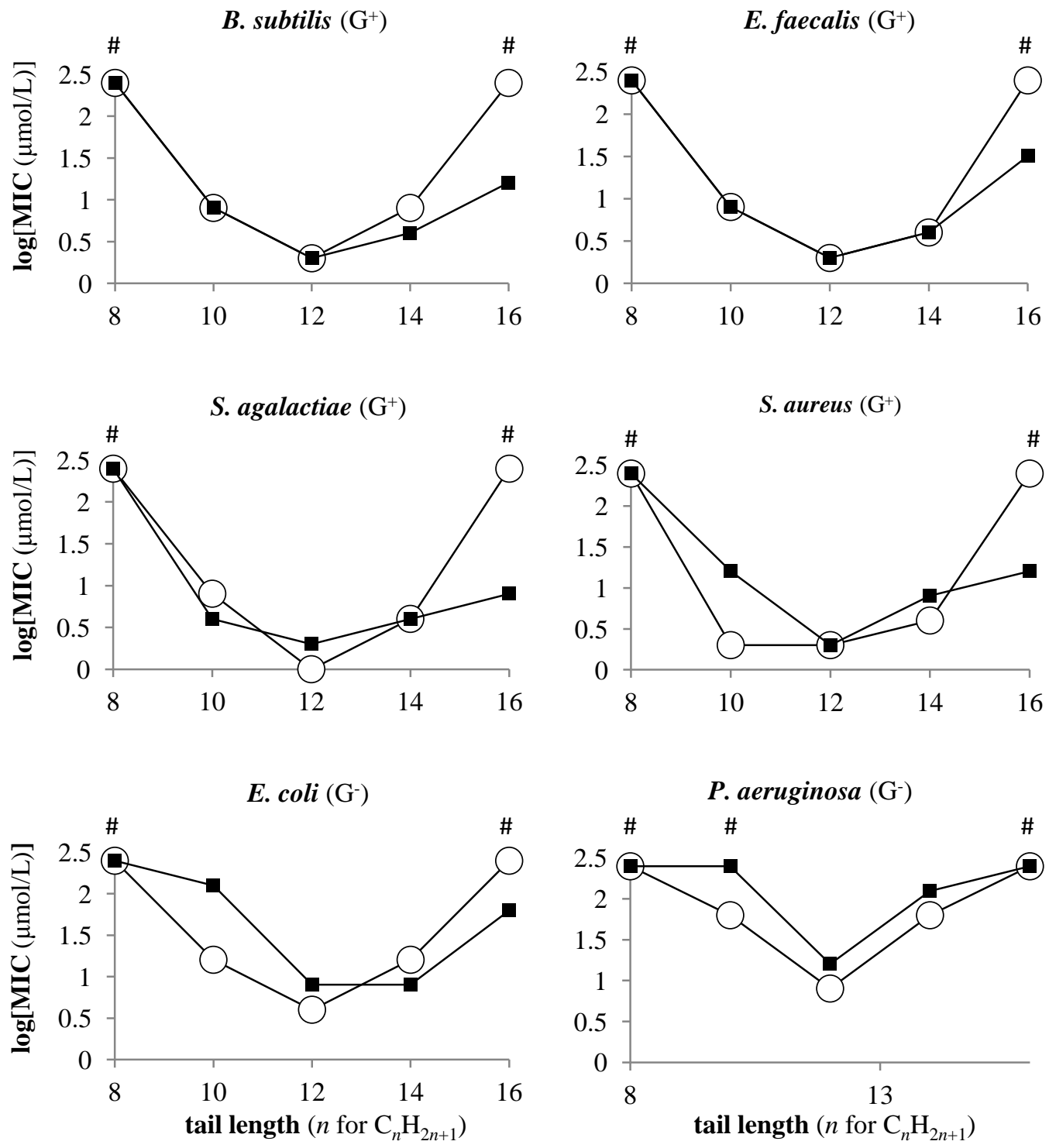

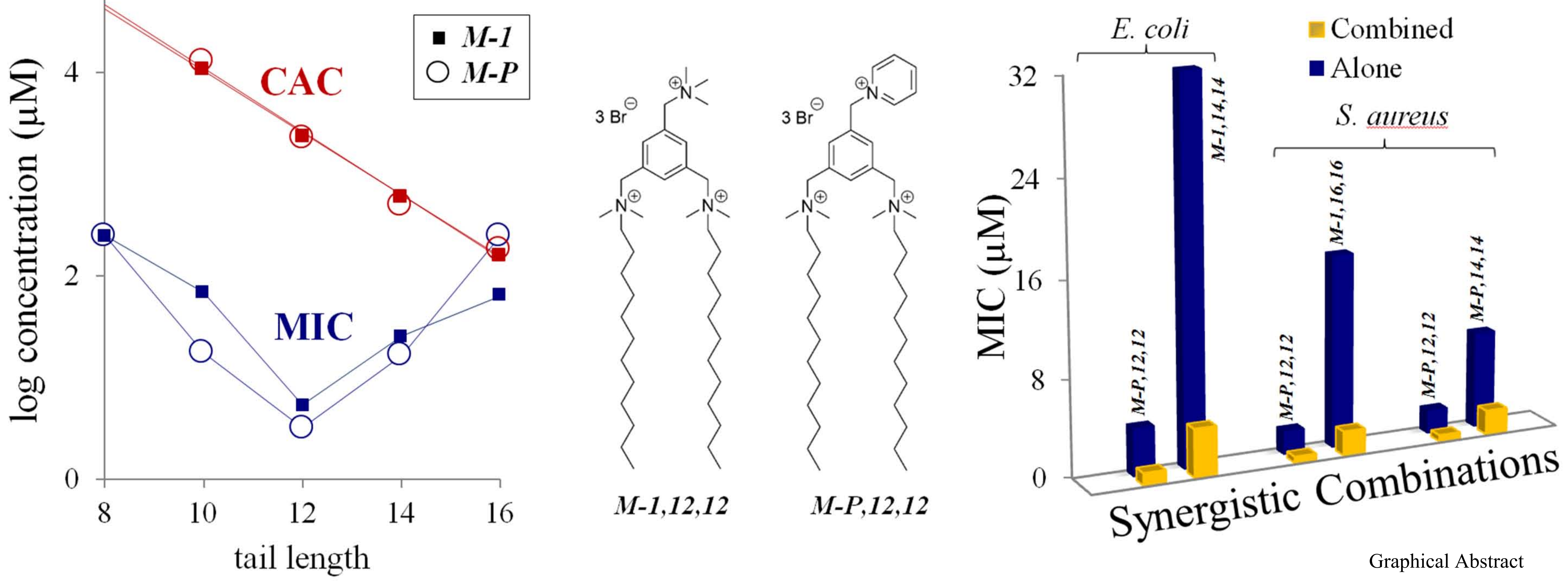